Draft version August 7, 2018

Typeset using $\mathrm{LAT}_{\mathrm{E}} \mathrm{X}$ twocolumn style in AASTeX61

\title{
X-RAY LUMINOSITY AND SIZE RELATIONSHIP OF SUPERNOVA REMNANTS IN THE LMC
}

\author{
Po-Sheng Ou (歐柏昇), ${ }^{1,2}$ You-Hua Chu (朱有花), ${ }^{1,2,3}$ Pierre Maggi, ${ }^{4}$ Chuan-Jui Li (李傳睿), ${ }^{1,2}$ \\ Un PANG Chang (曾遠鵬), ${ }^{2}$ ANd Robert A. GruendL ${ }^{3,5}$
}

\author{
${ }^{1}$ Institute of Astronomy and Astrophysics, Academia Sinica, P.O. Box 23-141, Taipei 10617, Taiwan, R.O.C. \\ psou@asiaa.sinica.edu.tw \\ ${ }^{2}$ Department of Physics, National Taiwan University, Taipei 1061\%, Taiwan, R.O.C. \\ ${ }^{3}$ Department of Astronomy, University of Illinois at Urbana-Champaign, 1002 West Green Street, \\ Urbana,IL 61801, U.S.A. \\ ${ }^{4}$ Département d'Astrophysique, IRFU, CEA, Université Paris-Saclay, F-91191 Gif-sur-Yvette, France. \\ ${ }^{5}$ National Center for Supercomputing Applications, 1205 West Clark St., Urbana, IL 61801, U.S.A.
}

The Large Magellanic Cloud (LMC) has 60 confirmed supernova remnants (SNRs). Because of the known distance, $50 \mathrm{kpc}$, the SNRs' angular sizes can be converted to linear sizes, and their X-ray observations can be used to assess X-ray luminosities $\left(L_{X}\right)$. We have critically examined the LMC SNRs' sizes reported in the literature to determine the most plausible sizes. These sizes and the $L_{X}$ determined from XMM-Newton observations are used to investigate their relationship in order to explore the environmental and evolutionary effects on the X-ray properties of SNRs. We find: (1) Small LMC SNRs, a few to $10 \mathrm{pc}$ in size, are all of Type Ia with $L_{X}>10^{36} \mathrm{ergs} \mathrm{s}^{-1}$. The scarcity of small core-collapse (CC) SNRs is a result of CCSNe exploding in the low-density interiors of interstellar bubbles blown by their massive progenitors during their main sequence phase. (2) Medium-sized (10-30 pc) CC SNRs show bifurcation in $L_{X}$, with the X-ray-bright SNRs either in an environment associated with molecular clouds or containing pulsars and pulsar wind nebulae and the X-ray-faint SNRs being located in low-density interstellar environments. (3) Large (size $>30 \mathrm{pc}$ ) SNRs show a trend of $L_{X}$ fading with size, although the scatter is large. The observed relationship between $L_{X}$ and sizes can help constrain models of SNR evolution.

Keywords: ISM: supernova remnants — supernovae: general — X-rays: ISM — Magellanic Clouds 


\section{INTRODUCTION}

Most supernova remnants (SNRs), regardless of progenitor types, exhibit some kind of X-ray emission. Thermal emission can arise from shocked interstellar medium (ISM) and/or SN ejecta, while relativisic electrons interacting with amplified magnetic field can produce non-thermal (synchrotron) emission. In the cases of core-collapse (CC) SNRs, there may exist additional $\mathrm{X}$-ray emission from pulsars and pulsar wind nebulae (PWNe). See Vink (2012) for a comprehensive review of X-ray emission from SNRs.

To make a statistical study of X-ray emission of SNRs, we need a large sample of SNRs with known distances. The Galactic sample of SNRs is quite incomplete because of heavy absorption in the Galactic plane, and the distances to individual SNRs are often very uncertain. The Large Magellanic Cloud (LMC), on the other hand, has small internal and foreground absorption column densities (Schlegel et al. 1998), and hosts a large sample of SNRs all at essentially the same known distance $50 \mathrm{kpc}^{1}$ (Pietrzyński et al. 2013). At least 59 SNRs have been confirmed and a few additional SNR candidates have been suggested (Maggi et al. 2016; Bozzetto et al. 2017). This large sample of LMC SNRs is ideal for systematic and statistical studies of X-ray emission from SNRs.

Recently, Maggi et al. (2016) analyzed XMM-Newton observations of the 59 confirmed SNRs in the LMC, deriving physical properties of the X-ray-emitting plasma from spectral fits. Because of the known distance, it is possible to determine the X-ray luminosity of each SNR. In the meantime, Bozzetto et al. (2017, hereafter Bo2017) measured the sizes of the 59 LMC SNRs using $\mathrm{X}$-ray, radio and optical images. Intrigued by these results, we have examined the relationship between X-ray luminosity and size of LMC SNRs in order to explore evolutionary effects and environmental impacts on Xray properties of SNRs.

This paper reports our investigation of the relationship between X-ray luminosity and size of SNRs in the LMC. In Section 2, we discuss the physical meaning of SNR sizes measured at optical or X-ray wavelengths, examine the SNR sizes reported in the literature, and assess the most reliable sizes that represent the SNRs' full extent. In Section 3, we plot X-ray luminosities against sizes for LMC SNRs and note intriguing fea-

\footnotetext{
1 Note that due to the LMC's inclination of 18-23 degrees in the line of sight, the error in the distance and linear size can be uncertain by up to $10 \%$ (Subramanian \& Subramaniam 2010), and the luminosity can be uncertain by $20 \%$. These uncertainties, however, do not affect the general conclusions of these paper.
}

tures in the distribution of SNRs in this plot. In Section 4 , we discuss the physical reasons behind the distribution of SNRs in the plot of X-ray luminosity versus size. Finally, a summary is given in Section 5.

\section{SIZES OF LMC SNRS}

Both X-ray and optical images have been used to measure SNR sizes, but it should be noted that while Xray and optical $\mathrm{H} \alpha$ emission both originate from postshock gas, they arise under different physical conditions. Generally speaking, X-ray emission comes from hot gas with temperatures $\gtrsim 10^{6} \mathrm{~K}$, while $\mathrm{H} \alpha$ emission originates from ionized gas at $\sim 10^{4} \mathrm{~K}$; therefore, an SNR size measured in X-rays may differ from that measured in $\mathrm{H} \alpha$.

Measurements of X-ray and $\mathrm{H} \alpha$ sizes of SNRs can also differ because of different instrumental sensitivities. For example, the XMM-Newton observations of the LMC SNRs detect volume emission measures $\left(E M_{V} \equiv\right.$ $\int n_{e} n_{H} d V$, where $n_{e}$ is the electron density, $n_{H}$ is the hydrogen density, and $V$ is the emitting volume) of $10^{54}$ $-10^{60} \mathrm{~cm}^{-3}$ (Maggi et al. 2016). For a spherical volume of highly ionized interstellar gas $\left(n_{e} / n_{H} \sim 1.2\right.$ for a typical helium to hydrogen number ratio of 0.1 ), the rms density derived from the volume emission measure is

$$
\left\langle n_{H}^{2}\right\rangle^{1 / 2}=\left(\frac{5 E M_{V}}{\pi f D^{3}}\right)^{1 / 2},
$$

where $D$ is the diameter of the X-ray emitting gas, and $f$ is the volume filling factor. For SN ejecta dominated by heavy elements, $n_{e} / n_{H}$ should be greater than 1.2 , and hence the rms hydrogen density in equation (1) is the upper limit, which is about $(0.001-3) f^{-1 / 2} \mathrm{~cm}^{-3}$ for the LMC SNRs as derived from the XMM-Newton measurements. Meanwhile, narrow-band $\mathrm{H} \alpha$ CCD images can easily detect emission measures $\left(E M_{\ell} \equiv \int n_{e} n_{H} d \ell\right.$, where $\ell$ is the emitting path length) down to $10-20 \mathrm{~cm}^{-6}$ pc, and for an emitting path length of 5 pc the electron density needs to be at least $1.4-2 \mathrm{~cm}^{-3}$. Thus, for SNRs running into a dense medium with densities $>1 \mathrm{H}$-atom $\mathrm{cm}^{-3}$, the shocked gas can be detected in both X-ray and optical wavelengths, while those running into a medium with densities $\ll 1 \mathrm{H}$-atom $\mathrm{cm}^{-3}$ may be visible in $\mathrm{X}$-rays but not in optical.

Another factor that can affect the measurements of SNR sizes is the wavelength-dependent confusion from the background. SNRs may be located adjacent to HII regions or superposed on a complex background, in which case the boundary of an SNR can be diagnosed by sharp filamentary morphology, enhanced [S II] line emission, high-velocity components in optical emission lines, nonthermal radio emission, and diffuse X-ray emission (Chu 1997). When more than one of the above diag- 

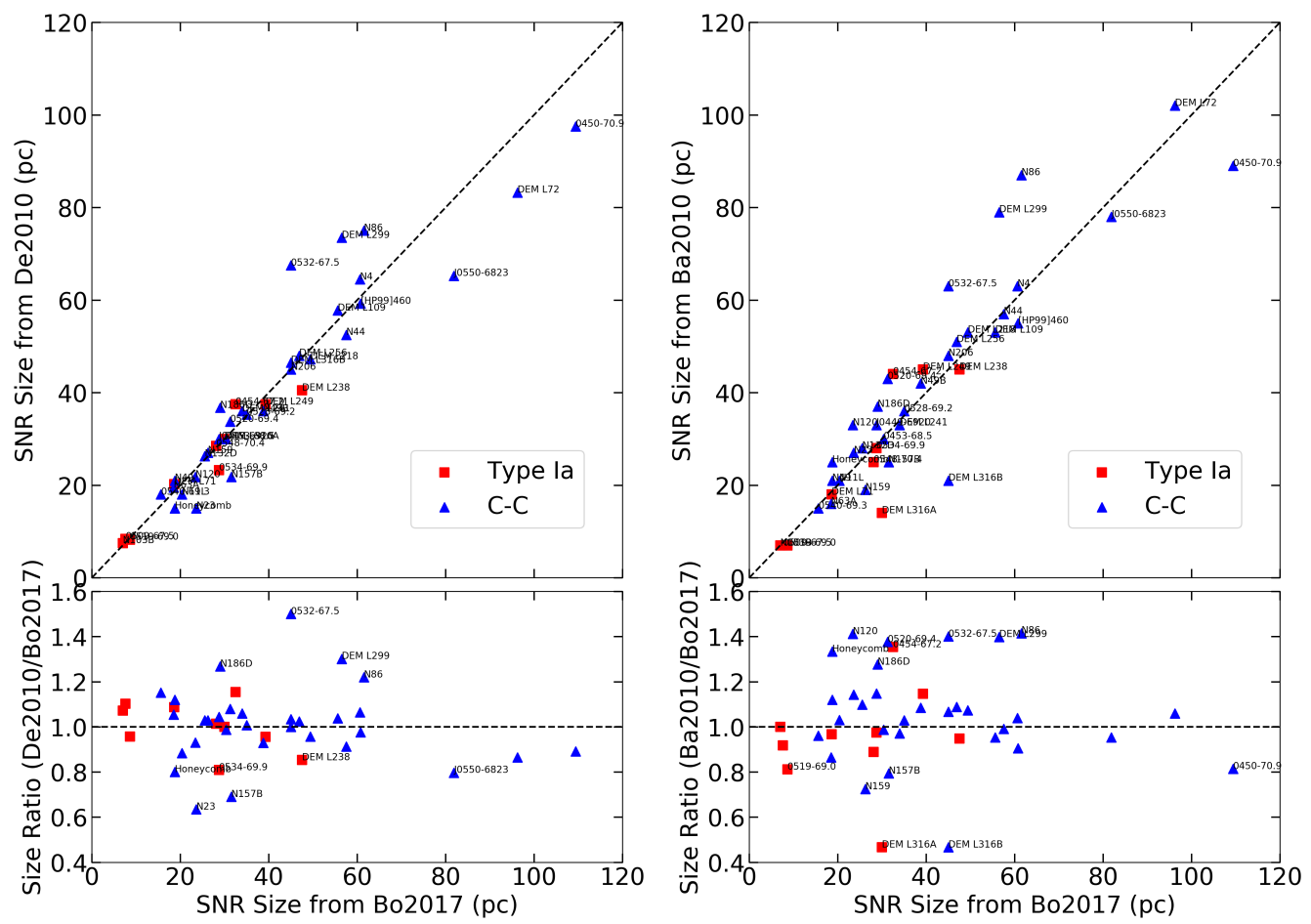

Figure 1. The left panels compare the SNR sizes reported by De2010 and Bo2017, with the upper panel plotting De2010 sizes versus Bo2017 sizes and the lower panel plotting the (De2010 size / Bo2017 size) ratios versus Bo2017 sizes. The right panels compare the SNR sizes reported by Ba2010 and Bo2017 in the same way as the left panels.

nostics are detected, the SNR boundary can be more reliably measured. However, if X-ray emission is the only diagnostic detected and the SNR emission is superposed on a large-scale diffuse X-ray emission, the background confusion can prevent accurate measurements of the SNR size.

Several publications have reported sizes of the LMC SNRs, but there are often discrepancies between their measurements. Badenes et al. (2010, hereafter Ba2010) used mainly X-ray images from Chandra or XMMNewton to determine the SNR sizes, and adopted previous optical and radio measurements when highresolution X-ray images were not available. Desai et al. (2010, hereafter De2010) considered optical and X-ray images, and measured SNR sizes based on the extent of diffuse X-ray emission or filamentary $\mathrm{H} \alpha$ shell structure. Bo2017 considered optical, X-ray and radio images. Maggi et al. (2016) also listed SNR sizes, but they only gave the maximal diameters in X-ray; thus these sizes are often much larger than the ones reported by the above three references. Below we compare the SNR sizes reported by Ba2010, De2010, and Bo2017.

Bo2017 has the largest and most complete SNR sample, and is thus chosen to be the reference for comparisons. Figure 1 compares SNR sizes reported by De2010 and Bo2017 in the left panels, and Ba2010 and Bo2017 in the right panels. The upper panels plot SNR sizes from one source versus another, while the lower panels plot the ratios of SNR sizes from two sources.

De2010 and Bo2017 both used primarily optical and X-ray images for the SNR size measurements, but there are still discrepancies greater than $16 \%$ and up to $50 \%$. The discrepancies are caused by the following reasons: (1) The SNR sizes can be measured only in X-rays and the surface brightness varies significantly, such as N23, or the background is complex, such as the Honeycomb and 0532-67.5; in such cases the discrepancy in size measurements can be as high as $50 \%$. (2) The SNR is superposed on an $\mathrm{H}$ II region or a superbubble, whose $\mathrm{H} \alpha$ emission can confuse the size measurements, such as N157B and N186D. (3) The SNR size is measured without simultaneously considering optical, $\mathrm{X}$-ray, and radio images that show wavelength-dependent distribution of emission, such as 0534-69.9, DEM L238, DEM L299, and J0550-6823. (4) The irregular shape of an SNR can cause subjective size measurements to differ by up to $\sim 20 \%$, such as N86. For these discrepant objects, we examine their $\mathrm{H} \alpha$, [S II], X-ray, and $24 \mu \mathrm{m}$ images (in Appendix A), consider radio and kinematic properties available in the literature, and make new measurements (described in Appendix B).

The comparisons between Ba2010 and Bo2017 sizes, right panels of Figure 1, show numerous large discrepancies. These discrepancies are caused by the larger 
uncertainties in Ba2010 sizes that were compiled from previous measurements based on mainly X-ray images and some optical images. As mentioned above and detailed in Appendices A-B, multi-wavelength examination of an SNR provides the most comprehensive picture of its physical structure and boundaries, and size measurements based on only one single wavelength may not reflect the SNR's true extent.

\section{RELATIONSHIP BETWEEN SIZES AND $L_{X}$}

The LMC provides an ideal sample of SNRs for us to study the relationship between their X-ray luminosities $\left(L_{X}\right)$ and sizes. The size of an SNR may be intuitively thought to reflect its evolutionary stage, because an SNR expands as the shock wave propagates outward and a large SNR would be older than a small SNR, if the ambient interstellar densities are similar. However, the ambient ISM does have a wide variety of physical properties and conditions, and the relationship between size and evolution can be quite complex. Through the relationship between $L_{X}$ and size we hope to investigate effects of ambient environment and evolution on the SNRs' X-ray luminosities.

SNRs rarely show round, symmetric shell structures with well-defined sizes. To assign a "size" to an irregular SNR, we adopt the average of its major and minor axes. Such SNR sizes determined from data of Ba2010, De2010, and Bo2017 are tabulated in Appendix C. As discussed at length in Section 2, De2010 and Bo2017 sizes were determined primarily with optical and X-ray images of SNRs and are in agreement for most cases. For $83 \%$ of the SNRs that have Bo2017 and De2010 sizes differ by less than $16 \%$, we adopt their average sizes from Bo2017. For the SNRs with larger discrepancies between De2010 and Bo2017, we discuss individual objects and determine their average sizes in Appendix B, and list their adopted sizes in the table in Appendix C.

The LMC sample of SNRs have been studied in Xrays with XMM-Newton by Maggi et al. (2016). They fit the X-ray spectra with the package XSPEC (Arnaud 1996) using a combination of collisional ionization equilibrium (CIE) models and non-equilibrium ionization (NEI) models, derived their X-ray fluxes in the 0.3 to $8 \mathrm{keV}$ band, and computed their $L_{X}$ for an LMC distance of $50 \mathrm{kpc}$ (Pietrzyński et al. 2013). These observed (absorbed) $L_{X}$ are listed in the last column of the Table in Appendix C.

Using the $L_{X}$ and average sizes in Appendix C, we plot the $L_{X}$ versus size for the LMC SNRs in Figure 2. We have also made the same $L_{X}$-size plot with unabsorbed $L_{X}$ and present it in Figure 3. (These unabsorbed $L_{X}$ are from the same model fits that produced the absorbed
$L_{X}$ published by Maggi et al. 2016). The distribution of the SNRs are qualitatively similar to that in Figure 2. Note that we did not include SN 1987A ( $\operatorname{size}=0.45$ pc, $L_{X}=2.7 \times 10^{36} \mathrm{ergs} \mathrm{s}^{-1}$ ) because its inclusion will leave vast empty space on the left and compress all the data points on the right in Figure 2 and 3.

At first glance, the $L_{X}$ - size plot for LMC SNRs shows a scattered diagram; however, if the sizes are divided into three ranges: $<10 \mathrm{pc}$ as "small", $10-30 \mathrm{pc}$ as "medium", and $>30 \mathrm{pc}$ as "large", it is possible to see interesting trends in each size range:

(1) For sizes a few to $10 \mathrm{pc}$, only a small group of SNRs exist with $L_{X}$ of a few $\times 10^{36} \mathrm{ergs} \mathrm{s}^{-1}$, and all of them are of Type Ia. For comparison, we add the Galactic SNRs with sizes a few to $10 \mathrm{pc}$ in Figure 3; the data are taken from the Chandra Supernova Remnant Catalog ${ }^{2}$. Interestingly, Tycho and Kepler SNRs, two small Type Ia SNRs in our Galaxy, are also located in the similar part of $L_{X}$-size plot as the small LMC Type Ia SNRs. In contrast, the Galactic CC SNR Cas A is an order of magnitude more luminous than these small Type Ia SNRs, and the 100 -year old Galactic Type Ia SNR G1.9+0.3 is smaller and significantly fainter (Reynolds et al. 2008; Borkowski et al. 2010, 2013).

(2) For sizes 10-30 pc, there is a bifurcation in the distribution of SNRs. The X-ray-bright ones have $L_{X}>10^{36}$ $\operatorname{ergs~s}^{-1}$ and the X-ray-faint ones have $L_{X}<10^{35} \mathrm{ergs}$ $\mathrm{s}^{-1}$ for sizes below $\sim 20 \mathrm{pc}$, and these two groups converge to a few $\times 10^{35} \mathrm{ergs} \mathrm{s}^{-1}$ towards $30 \mathrm{pc}$ size. It is worth noting that the X-ray-faint medium-sized SNRs are mostly CC SNRs.

(3) For sizes $>30 \mathrm{pc}$, while $L_{X}$ exhibits a wide range, the majority of the SNRs appear to show a general trend of $L_{X}$ decreasing with size.

It also appears that the Type Ia SNRs show smaller scatter in $L_{X}$ for any given size than the CC SNRs, especially in the medium size range (Figures 2 and 3 ). The scatter in $L_{X}$ reflects the ambient interstellar density: Type Ia SNe occur in diffuse medium with moderate densities, while CC SNe can take place near dense molecular clouds or in a very low-density environment produced by energy feedback from massive stars. Because of the smaller scatter in $L_{X}$, the smooth variations of Type Ia SNRs' $L_{X}$ versus size may demonstrate the SNR evolution.

The dashed line in the lower right corner of Figure 2 corresponds to a constant surface brightness of $10^{29}$ $\operatorname{ergs~s}^{-1} \operatorname{arcsec}^{-2}$, which represents the typical detection limit of the XMM-Newton observations used by Maggi et

\footnotetext{
${ }^{2}$ See http://hea-www.cfa.harvard.edu/ChandraSNR/.
} 


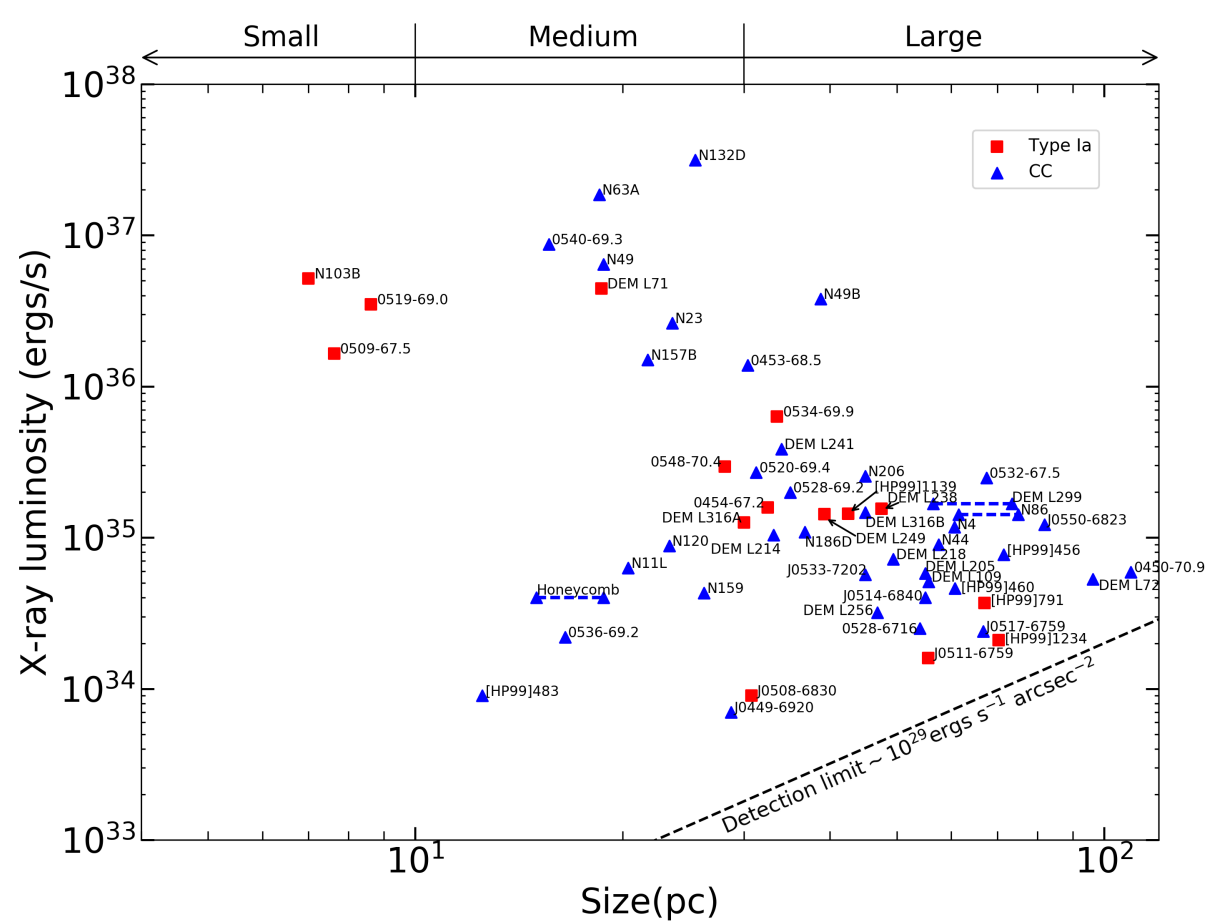

Figure 2. Observed $L_{X}$ versus size plot for SNRs in the LMC. The size and $L_{X}$ are listed in Appendix C. For the Honeycomb SNR and N86, we have used two extreme size measurements to illustrate the largest uncertainties in the size measurements. Note that some of the large CC SNRs may in fact be Type Ia SNRs whose SN ejecta have cooled and no longer emit detectable $\mathrm{X}$-rays for them to be identified as such.

al. (2016). Consequently, no SNRs are located beneath this dashed line.

\section{DISCUSSION}

We have examined the physical structures and environments of SNRs in the three size ranges in order to understand the physical significance of their distributions in the $L_{X}$-size plot. The discussion in this section is ordered according to the SNR sizes.

\subsection{Small Known LMC SNRs Are Dominated by Type Ia}

It is striking that the small LMC SNRs, with sizes a few to $10 \mathrm{pc}$, are all Type Ia SNRs with $L_{X}$ of a few $\times$ $10^{36}$ ergs s $^{-1}$. (Note that SN 1987A is outside the size range under discussion.) For comparison, we show that the Galactic Type Ia SNRs Kepler and Tycho are both located in a similar region as the young LMC Type Ia SNRs. The small range of $L_{X}$ for small Type Ia SNRs and the scarcity of small CC SNRs can be explained as follows.

Type Ia SNe are usually considered to explode in a tenuous and uniform ISM (e.g., Badenes et al. 2005). On the other hand, CC SNe usually explode inside interstellar bubbles blown by the fast stellar winds of their mas- sive progenitors during the main sequence phase (Castor et al. 1975; Weaver et al. 1977). Interstellar bubble interiors have very low densities, and hence CC SNe inside bubbles are called "cavity explosions". It is conceivable that the interstellar environments of Type Ia and CC SNe have very different density profiles.

Density profiles of ambient medium strongly affect the evolution of an SNR's $L_{X}$. In a classical model of a SN explosion in a uniform ISM, the resulting SNR goes through free expansion phase, Sedov phase (i.e., adiabatic phase), and radiative phase (Woltjer 1972). The Sedov phase starts when the swept-up ISM mass is several times the SN ejecta mass (e.g., Dwarkadas \& Chevalier 1998). The $L_{X}$ of an SNR during the Sedov phase can be calculated (e.g., Hamilton et al. 1983). To illustrate the evolution of $L_{X}$ for different ambient densities, we plot $L_{X}$ against age and size in Figure 4.

For a Type Ia SNR in a partially neutral ISM, only the ionized interstellar gas can be swept up by the shock. Thus, for a uniform density of $\sim 1 \mathrm{H}$-atom $\mathrm{cm}^{-3}$ and a neutral fraction of $\eta$, the Sedov phase will start when the swept-up ionized gas reaches $1.4 M_{\odot}$ in mass, corresponding to a radius of $2.4(1-\eta)^{-1 / 3}$ pc. This radius is $5.2 \mathrm{pc}$ if $\eta=0.9$, and $3 \mathrm{pc}$ if $\eta=0.5$. These sizes are comparable to the young Balmer-dominated Type 


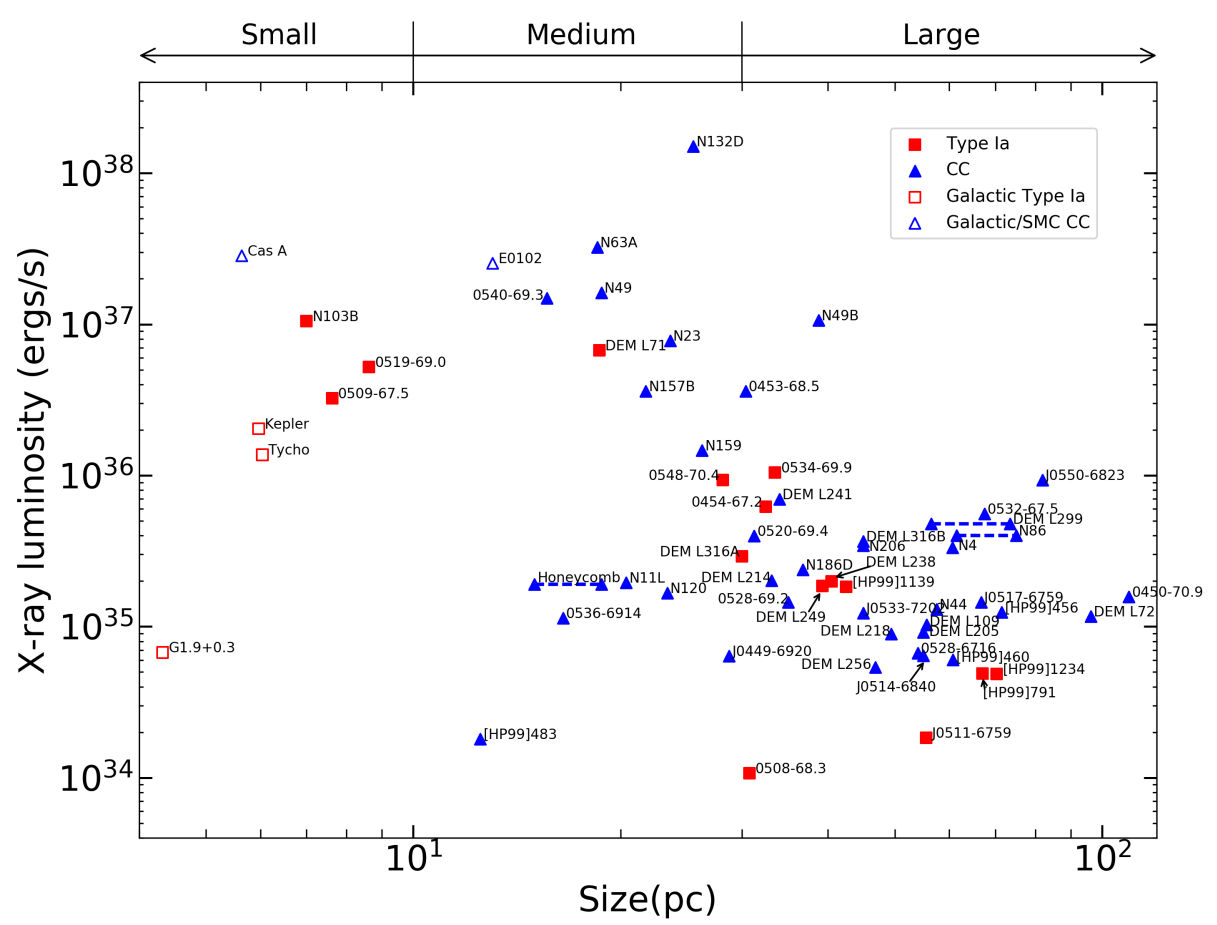

Figure 3. Unabsorbed $L_{X}$ versus size plot for SNRs in the LMC.
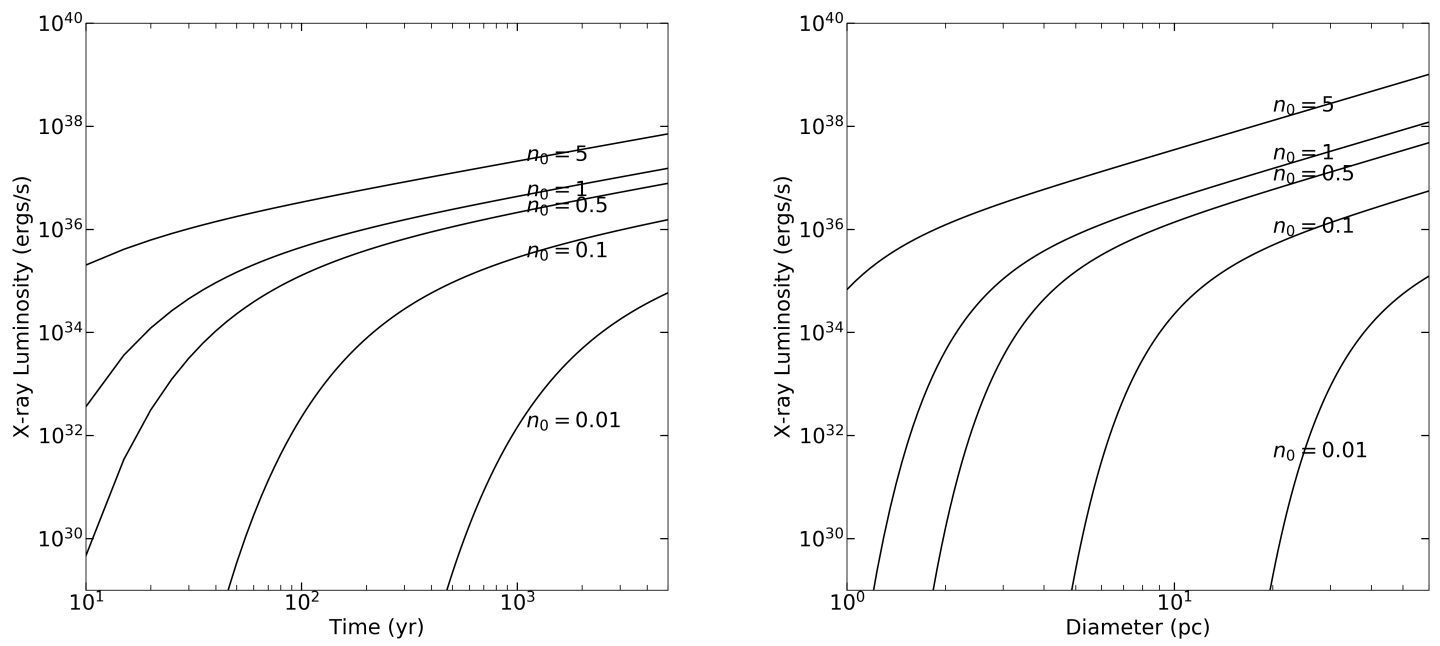

Figure 4. Evolution of $L_{X}$ in the Sedov model. The ambient density $n_{0}$ in H-atom $\mathrm{cm}^{-3}$ is marked for each model.

Ia SNRs in the LMC, 0509-67.5 and 0519-69.0; thus, it is likely that these young Type Ia SNRs are entering the Sedov phase. However, the interstellar density is so much lower than the SN ejecta density that their X-ray emission is still dominated by that produced by the reverse shock into the SN ejecta. This is evidenced in the $\mathrm{SN}$ ejecta abundance revealed in the X-ray spectra of these small Balmer-dominated Type Ia SNRs, although the X-ray emission shows a shell morphology (Warren \& Hughes 2004; Kosenko et al. 2010). The larger Type Ia SNRs, such as DEM L71 and 0548-70.4 with sizes in the 20-30 pc range, must be in the Sedov phase already. Furthermore, their forward shock and reverse shock have traveled farther apart, and their X-ray emission shows 
the forward shock in an interstellar shell well resolved from the reverse shock in the SN ejecta (Hughes et al. 2003; Hendrick et al. 2003).

$\mathrm{X}$-ray emission from reverse shocks is the cause of the high $L_{X}$ of small Type Ia SNRs. The small scatter of these young bright Type Ia SNRs in the $L_{X}$ vs size plot reflects their similar ages, the relative uniformity of $\mathrm{SNe}$ Ia (in term of nucleosynthesis and explosion energy), and the modest effect the progenitors have on changing their immediate surrounding. The smallest Galactic Type Ia SNR G1.9+0.3 has a low $L_{X}$ because it is so young $(<200 \mathrm{yr})$ that the reverse shock has only gone through very little of the SN ejecta (Reynolds et al. 2008; Borkowski et al. 2014).

For CC SNRs whose SNe exploded in cavities of windblown bubbles, due to the extremely low density within the bubbles $\left(\sim 10^{-4}-10^{-2} \mathrm{H}\right.$-atom $\left.\mathrm{cm}^{-3}\right)$, the Xray emission from shocked gas would be too faint to be detected at a young age; only when the SNR's forward shock hits the dense shell/wall of a bubble will the X-ray luminosity jump up several orders of magnitude (Dwarkadas 2005). As shown by Nazé et al. (2001), main sequence $\mathrm{O}$ stars have interstellar bubbles of sizes 15-20 pc. By the time a massive star explodes as a CC SN, its main-sequence bubble has grown larger, and hence the SNR shock goes through the low-density bubble interior without producing detectable X-ray emission until it hits the bubble shell wall at radius of $10 \mathrm{pc}$ or larger.

For illustration, considering a spherical interstellar bubble with a radius of $10 \mathrm{pc}$ and assuming a simplistic extreme case (upper limit) of average density of 0.01 $\mathrm{H}$-atom $\mathrm{cm}^{-3}$ in the bubble interior, we can calculate the total mass in the bubble interior to be $\lesssim 1 M_{\odot}$; thus, when the SNR shock reaches the bubble wall, it has swept up only $\sim 1 M_{\odot}$, much lower than the CCSN ejecta mass, a few to a few tens $M_{\odot}$; thus, the Sedov phase has not been reached. The bubble shell consists of swept-up ISM that was originally distributed in the bubble cavity. Assuming the bubble was blown in a diffuse ISM with density of $1 \mathrm{H}$-atom $\mathrm{cm}^{-3}$, the total mass in the bubble shell would be $100 M_{\odot}$; therefore, the SNR reaches the Sedov phase when the forward SNR shock traverses the bubble shell.

During the free-expansion phase, the SNR shock is not significantly decelerated and it remains fast until it hits the bubble wall. Assuming a constant shock velocity of $10,000 \mathrm{~km} \mathrm{~s}^{-1}$, it only takes 1000 years for the SNR to grow to a radius of $10 \mathrm{pc}$. Consequently, SNRs inside interstellar bubbles not only emit very faintly in X-rays, but also expand very rapidly to reach the dense shell wall. Such "cavity explosions" explain the absence of small CC SNRs in the $L_{X}$-size plot. Cavity explosions are also responsible for the discrepancies between ionization ages and dynamical ages of LMC SNRs, such as N132D, N63A, and N49B (Hughes et al. 1998).

We have plotted the young CC SNR Cas A in Figure 3 for comparison. Cas A is small in size and luminous in X-rays. These properties are caused by its interaction with a dense circumstellar medium, i.e., material ejected by the SN progenitor (Fesen 2001). Circumstellar bubbles are often observed around Wolf-Rayet stars and luminous blue variables (LBVs), and circumstellar bubbles are smaller than interstellar bubbles (Chu 2003). Cas A $\mathrm{SN}$ must have exploded in a circumstellar bubble.

\subsection{X-ray-Bright and X-ray-Faint Medium-Sized SNRs}

The medium-sized LMC SNRs show clear bifurcation in their $L_{X}$. In the X-ray-bright group with $L_{X} \geq 10^{36}$ ergs $\mathrm{s}^{-1}$, only one is of Type Ia, and the other seven are CC SNRs. Among these X-ray-bright CC SNRs, four are interacting with molecular clouds, as $\mathrm{CO}$ emission was detected near the SNRs N23, N49, and N132D (Banas et al. 1997; Park et al. 2003) and $\mathrm{H}_{2}$ absorption is detected in Spitzer IRS observations towards N63A (Segura-Cox et al. 2018, in preparation). None of these four X-ray-bright CC SNRs show sharp $\mathrm{H} \alpha$ shell structure enclosing the diffuse $\mathrm{X}$-ray emission, indicating that the forward SNR shocks are still in the lowdensity interiors of bubbles. In the cases of N23 and N132D, where no prominent shocked cloudlets are seen, the X-ray emission does show limb-brightening, indicating that the ambient medium is dense enough to produce detectable X-ray emission but not optical $\mathrm{H} \alpha$ emission, and this ambient medium may correspond to the conduction layer in a bubble interior (Weaver et al. 1977). As N23 and N132D are both associated with molecular clouds, their bubble shells and conduction layers must have higher densities, which contribute to the bright Xray emission. In the cases of N49 (Bilikova et al. 2007; Park et al. 2012) and N63A (Warren et al. 2003), it is clear that dense cloudlets, possibly associated with the molecular clouds, have been shocked and contribute to the X-ray emission. The other three X-ray-bright CC SNRs possess bright PWNe: 0540-69.3 (Gotthelf \& Wang 2000), N157B (Wang \& Gotthelf 1998), and 0453-68.5 (Gaensler et al. 2003). Pulsars and PWNe are powerful sources of nonthermal X-ray emission and provide additional X-ray emission to boost their SNRs' total $L_{X}$. Note that the PWN of $0453-68.5$ is not particularly dominating, but its X-ray image show a limbbrightened sharp shell that indicates that the shock has already reached the bubble shell. While 0453-68.5 has 
a PWN, it is the SNR shock impact on the dense bubble shell giving rise to $L_{X}$.

The X-ray-faint medium-sized SNRs are mostly associated with $\mathrm{CC} \mathrm{SNe}$. Among the three X-ray-faint CC SNRs smaller than 20 pc, 0536-69.2 and [HP99]483 are not detected in optical, and the Honeycomb SNR shows only a small patch of honeycomb-like nebulosity resulting from SNR shocking a piece of shell wall (Chu et al. 1995; Meaburn et al. 2010). The absence of sharp optical shells enclosing the diffuse X-ray emission indicates a low-density ISM around these SNRs. The Honeycomb SNR has hit a small piece of dense gas and hence it has the highest $L_{X}$ among these three, but still a couple orders of magnitude fainter than the SNRs interacting with molecular clouds. The X-ray-faint SNRs with sizes $20-30$ pc all show optical shell structure enclosing their diffuse X-ray emission, and they have higher $L_{X}$ than the smaller ones, except J0449-6920, whose $X M M$-Newton observation was too shallow to make accurate measurements. These CC SNRs may represent cavity explosions whose SNR shocks have just reached the bubble shell walls. The SNRs N11L and N120 have just reached the bubble shell, but the bubble shell densities are not as high as those of N23 and N132D.

\subsection{Fading of $X$-rays in Large SNRs}

Among the large (size $>30 \mathrm{pc}$ ) LMC SNRs, a general trend of decreasing $L_{X}$ for larger SNRs can be seen, but for any given size, the differences in $L_{X}$ can be up to one order of magnitude.

As an SNR sweeps up more interstellar gas, the shock velocity decreases and when it goes much below $\sim 300$ $\mathrm{km} \mathrm{s}^{-1}$, the post-shock temperature will be below $10^{6} \mathrm{~K}$, too low to generate $\mathrm{X}$-ray-emitting gas. The hot gas in SNR interior cools, and the X-ray emission diminishes.

The scatter in $L_{X}$ may be caused by the differences in ambient gas densities $\left(n_{0}\right)$ and the $\mathrm{SN}$ explosion energies $(E)$. To evaluate the effects of these two factors, we consider a spherical SNR of radius $R$, whose X-ray emission originates from shocked ISM in a shell. Its $L_{X}$ is $\propto$ (emitting volume $) \times(\text { density })^{2} \times($ emissivity $)$. As (emitting volume) $\times$ (density) is proportional to the total interstellar mass within radius $R$, it is $\propto R^{3} n_{0}^{2}$. The emissivity is a slow function of temperature for photon energies below $2 \mathrm{keV}$ (Hamilton et al. 1983). Since the large old SNRs are likely at low X-ray emitting temperatures, a few $\times 10^{6} \mathrm{~K}$ at most, we will treat the emissivity as a constant, and $L_{X} \propto R^{3} n_{0}^{2}$.

The total kinetic energy in the SNR shell scales with the explosion energy, so $E \propto R^{3} n_{0} v^{2}$. The large old SNRs have low expansion velocities of a few $\times 10^{2} \mathrm{~km}$ $\mathrm{s}^{-1}$, so we will also approximate the expansion velocity as a constant. Thus, $L_{X} \propto E n_{0}{ }^{3}$. The effects of the ambient density and the SN explosion energy are about equally important. However, the ranges of the ambient gas densities and the SN explosion energies are quite different. The ambient interstellar density can range from 0.01 to a few hundred $\mathrm{H}$-atom $\mathrm{cm}^{-3}$, about 4 orders of magnitude, while the SN explosion energies are mostly clustered around $10^{51}$ ergs with extreme values differing by no more than 3 orders of magnitude (e.g., Woosley \& Weaver 1986).

Hence, the large scatter in $L_{X}$ for SNRs with the same size is more likely caused by the detailed differences in the ambient gas densities, and the SN explosion energy plays a lesser role in raising the scatter in $L_{X}$.

\section{SUMMARY}

The LMC is at a known distance of $50 \mathrm{kpc}$, and thus the linear sizes of SNRs in the LMC can be determined from their angular size measurements (Desai et al. 2010; Bozzetto et al. 2017), and their X-ray luminosities can be determined from XMM-Newton X-ray observations (Maggi et al. 2016), allowing a unique opportunity to examine the relationship between $L_{X}$ and size of SNRs. We have critically compared LMC SNR sizes reported by different authors in the literature and adopted the most reasonable sizes to investigate how $L_{X}$ vary with sizes among the LMC sample of SNRs.

We find that the $L_{X}$ - size relationship for LMC SNRs can be divided into small, medium, and large size ranges: (1) The small LMC SNRs with sizes a few to $10 \mathrm{pc}$ are all young Type Ia SNRs with $L_{X}$ a few times $10^{36} \mathrm{ergs}$ $\mathrm{s}^{-1}$. The apparent scarcity of small CC SNRs may be caused by their "cavity explosions", as massive progenitors of CCSNe have blown interstellar bubbles and the SN explosions take place in the very low-density interiors of the bubbles.

(2) The medium-sized SNRs, with sizes 10-30 pc, show bifurcation in their $L_{X}$ with an order of magnitude difference in $L_{X}$. The X-ray-bright CC SNRs either are in an environment associated with molecular clouds or have pulsars and pulsar-wind nebulae emitting nonthermal X-ray emission.

(3) The large SNRs, with sizes greater than $\sim 30 \mathrm{pc}$, show a general trend of fading $L_{X}$ at large sizes. As these sizes are larger than the normal interstellar bubbles blown by massive stars, the large SNRs have swept up the bubble material and extended into the diffuse

\footnotetext{
3 Note that this is in interesting contrast with the radio luminosity, which scales as $L_{\text {radio }} \propto E^{1.3} n_{0}^{0.45}$ or $L_{\text {radio }} \propto E^{1.45} n_{0}^{0.3}$ depending on the magnetic field amplification mechanism by the shock (Chomiuk \& Wilcots 2009).
} 
ISM. As the SNR shocks sweep up more ISM, the shock velocity slows down. When the post-shock velocities are too low to produce X-ray-emitting material, the hot plasma in SNR interiors cool and reduce the X-ray emission.
This project is supported by Taiwanese Ministry of Science and Technology grant MOST 104-2112-M-001044-MY3.

\section{REFERENCES}

Arnaud, K. A. 1996, Astronomical Data Analysis Software and Systems V, 101, 17

Badenes, C., Maoz, D., \& Draine, B. T. 2010, MNRAS, 407, 1301

Badenes, C., Borkowski, K. J., \& Bravo, E. 2005, ApJ, 624, 198

Banas, K. R., Hughes, J. P., Bronfman, L., \& Nyman, L.-Å. 1997, ApJ, 480, 607

Bilikova, J., Williams, R. N. M., Chu, Y.-H., Gruendl, R. A., \& Lundgren, B. F. 2007, AJ, 134, 2308

Borkowski, K. J., Reynolds, S. P., Green, D. A., et al. 2014, ApJL, 790, L18

Borkowski, K. J., Reynolds, S. P., Hwang, U., et al. 2013, ApJL, 771, L9

Borkowski, K. J., Reynolds, S. P., Green, D. A., et al. 2010, ApJL, 724, L161

Bozzetto, L. M., Filipović, M. D., Vukotić, B., et al. 2017, ApJS, 230, 2

Bozzetto, L. M., Filipovic, M. D., Crawford, E. J., et al. 2012, RMxAA, 48, 41

Castor, J., McCray, R., \& Weaver, R. 1975, ApJL, 200, L107

Chomiuk, L., \& Wilcots, E. M. 2009, ApJ, 703, 370

Chu, Y.-H. 2003, A Massive Star Odyssey: From Main Sequence to Supernova, 212, 585

Chu, Y.-H. 1997, AJ, 113, 1815

Chu, Y.-H., Dickel, J. R., Staveley-Smith, L., Osterberg, J., \& Smith, R. C. 1995, AJ, 109, 1729

Chu, Y.-H., Kennicutt, R. C., Jr., Schommer, R. A., \& Laff, J. 1992, AJ, 103, 1545

Desai, K. M., Chu, Y.-H., Gruendl, R. A., et al. 2010, AJ, 140,584

Dwarkadas, V. V. 2005, ApJ, 630, 892

Dwarkadas, V. V., \& Chevalier, R. A. 1998, ApJ, 497, 807

Fesen, R. A. 2001, ApJS, 133, 161

Gaensler, B. M., Hendrick, S. P., Reynolds, S. P., \& Borkowski, K. J. 2003, ApJL, 594, L111

Gotthelf, E. V., \& Wang, Q. D. 2000, ApJL, 532, L117

Hamilton, A. J. S., Sarazin, C. L., \& Chevalier, R. A. 1983, ApJS, 51, 115

Hendrick, S. P., Borkowski, K. J., \& Reynolds, S. P. 2003, ApJ, 593, 370

Hughes, J. P., Ghavamian, P., Rakowski, C. E., \& Slane, P. O. 2003, ApJL, 582, L95
Hughes, J. P., Hayashi, I., \& Koyama, K. 1998, ApJ, 505, 732

Kosenko, D., Helder, E. A., \& Vink, J. 2010, A\&A, 519, A11

Laval, A., Rosado, M., Boulesteix, J., et al. 1989, A\&A, 208, 230

Maggi, P., Haberl, F., Kavanagh, P. J., et al. 2016, A\&A, 585, A162

Meaburn, J., Redman, M. P., Boumis, P., \& Harvey, E. 2010, MNRAS, 408, 1249

Nazé, Y., Chu, Y.-H., Points, S. D., et al. 2001, AJ, 122, 921

Park, S., Hughes, J. P., Slane, P. O., et al. 2012, ApJ, 748, 117

Park, S., Burrows, D. N., Garmire, G. P., et al. 2003, ApJ, 586, 210

Pietrzyński, G., Graczyk, D., Gieren, W., et al. 2013, Nature, 495, 76

Reynolds, S. P., Borkowski, K. J., Green, D. A., et al. 2008, ApJL, 680, L41

Schlegel, D. J., Finkbeiner, D. P., \& Davis, M. 1998, ApJ, 500,525

Sedov, L. I. 1959, Similarity and Dimensional Methods in Mechanics, New York: Academic Press, 1959,

Subramanian, S., \& Subramaniam, A. 2010, A\&A, 520, A24

Taylor, G. 1950, Proceedings of the Royal Society of London Series A, 201, 159

Vink, J. 2012, A\&A Rv, 20, 49

Wang, Q. D., \& Gotthelf, E. V. 1998, ApJ, 494, 623

Warren, J. S., \& Hughes, J. P. 2004, ApJ, 608, 261

Warren, J. S., Hughes, J. P., \& Slane, P. O. 2003, ApJ, 583, 260

Warth, G., Sasaki, M., Kavanagh, P. J., et al. 2014, A\&A, 567, A136

Weaver, R., McCray, R., Castor, J., Shapiro, P., \& Moore, R. 1977, ApJ, 218, 377

Williams, R. M., Chu, Y.-H., Dickel, J. R., et al. 1999, ApJS, 123, 467

Woltjer, L. 1972, ARA\&A, 10, 129

Woosley, S. E., \& Weaver, T. A. 1986, ARA\&A, 24, 205 
APPENDIX

A. IMAGES OF THE SNRS WITH LARGE DISCREPANCIES BETWEEN DIFFERENT SIZE MEASUREMENTS
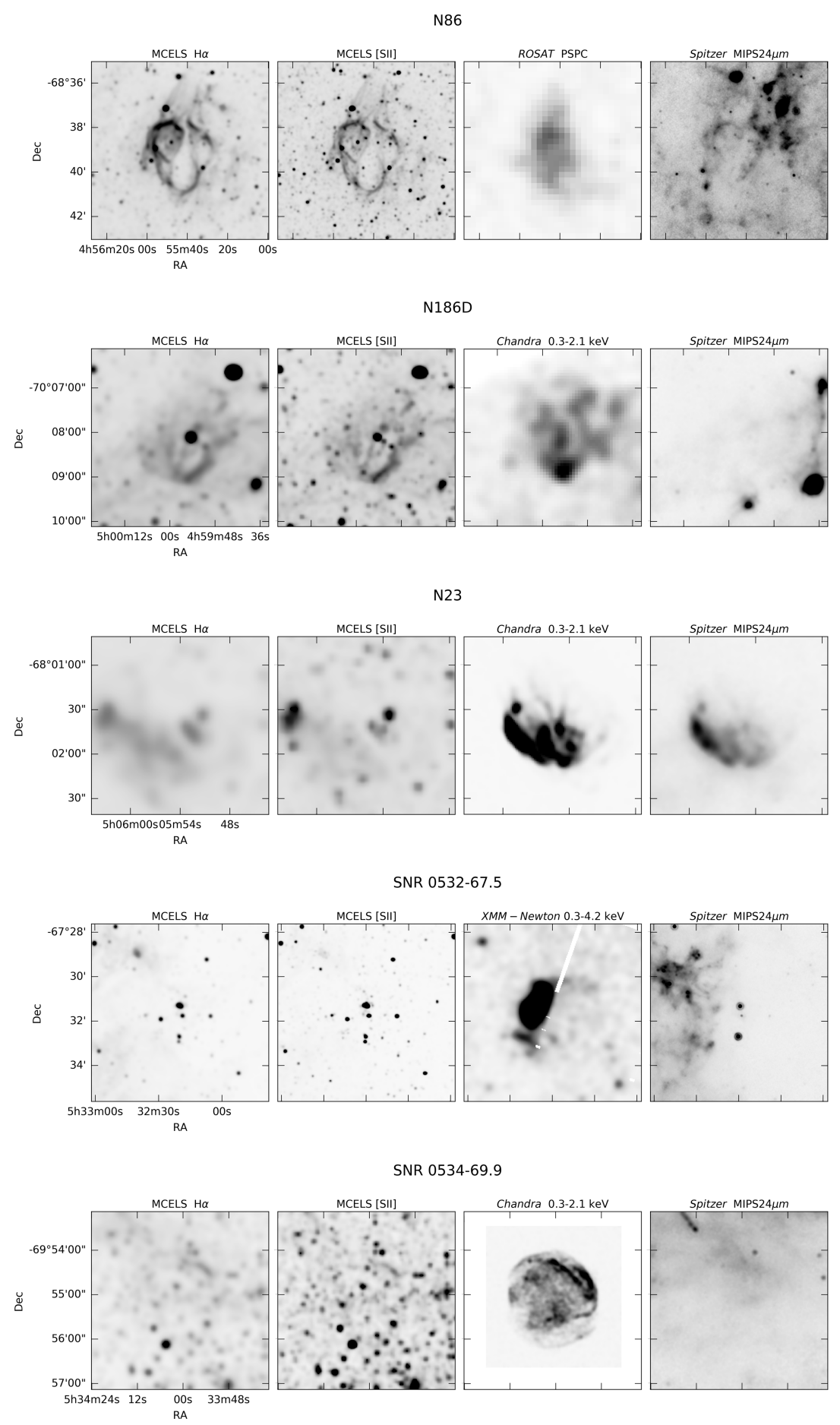

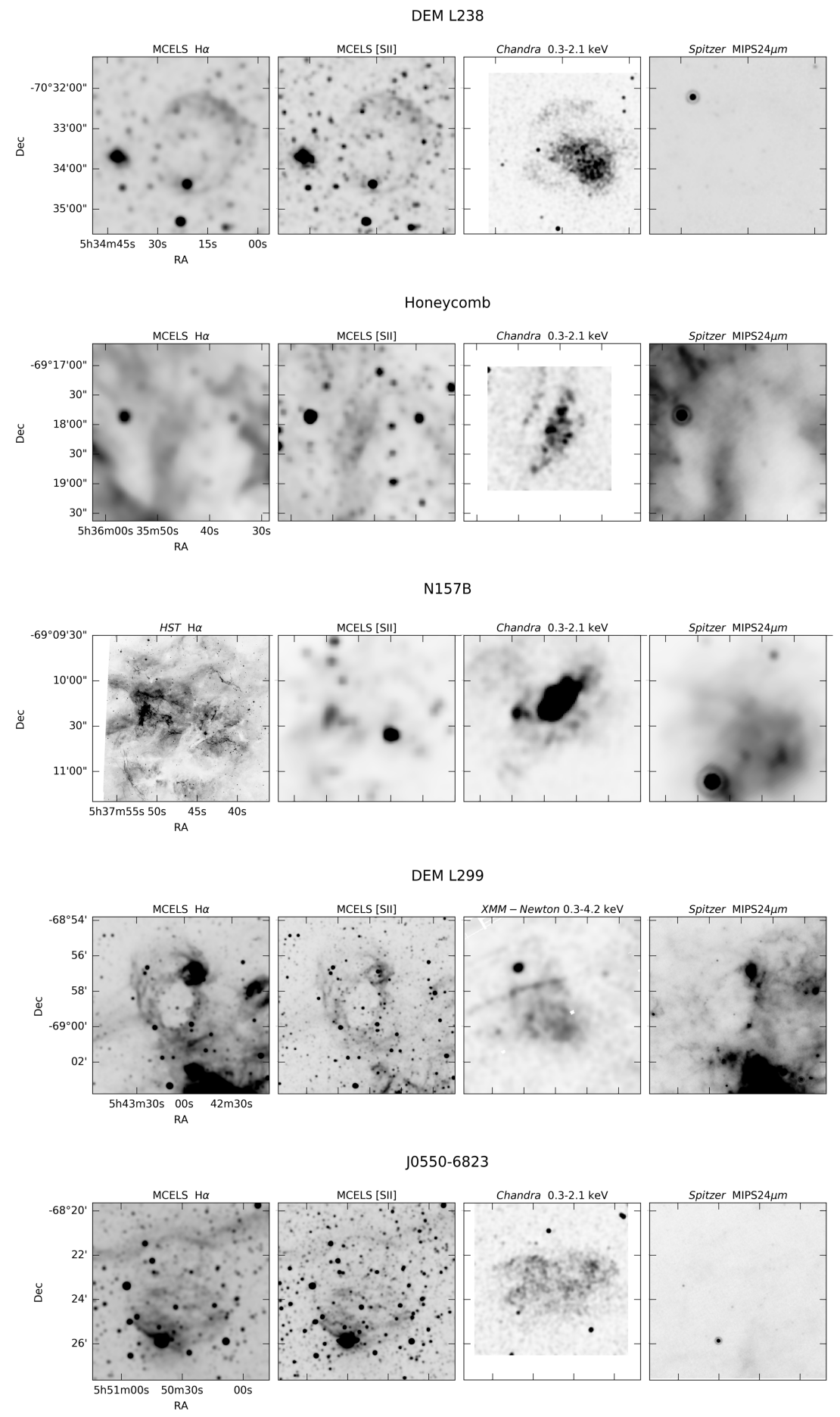

Figure 5. Images of the SNRs with large discrepancies between the size measurements of Bo2017 and De2010. 


\section{B. DESCRIPTIONS OF SIZE DETERMINATIONS FOR INDIVIDUAL SNRS}

N86.- The shape of the SNR is irregular because of the breakout structure in the north, through which the hot gas flows out from the SNR shell (Williams et al. 1999). For N86 in Figure 2, we have used both sizes of De2010 (75.0 pc, including the breakout) and Bo2017 (61.5 pc, not including the breakout) in the $L_{X}$ - size plot to illustrate the uncertainty in the size. Only low-resolution ROSAT X-ray images are available for N86; high-resolution Chandra and $X M M$-Newton observations will help refine the size determination.

N186D.- The size of SNR N186D in H $\alpha$ cannot be unambiguously measured, because N186D is projected on the rim of the superbubble N186E. Through the analysis of velocity fields in N186D, Laval et al. (1989) determined the SNR size to be $\sim 40 \mathrm{pc}$, which is consistent with the size of the [S II]-enhanced shell (see Figure 5 in Appendix A). Based on these considerations, we adopt the size 36.8 pc given by De2010.

N23.- X-ray emission of N23 is enhanced in the southeast side, likely due to a denser ambient medium (Williams et al. 1999). The size reported by De2010, $18 \times 12 \mathrm{pc}$, corresponds to only the X-ray-brightest region. Maggi et al. (2016) and Bo2017 included the fainter X-ray emission from the northwest side and reported a larger SNR size, 23.6 pc, which is more accurate and hence adopted in the $L_{X}$-size plot in Figure 2 .

SNR 0532-67.5.- This SNR may be associated with the OB association LH75 (Chu 1997). This SNR has no optical counterpart, indicating that it is in a low-density medium, possibly caused by the fast stellar winds and SN explosions from LH75. The size of this SNR can be measured only in X-rays. There is bright X-ray emission in a $\sim 40 \times 20 \mathrm{pc}$ region, and a fainter and larger X-ray arc connected with the bright region. As in the case of the SNR N23, we include both the bright and faint X-ray emission regions in the size estimate, and adopt a size of $67.5 \mathrm{pc}$ as the size of SNR 0532-67.5.

SNR 0534-69.9.- The optical images of this SNR show only a faint filament associated with the brightest X-ray emission region. Chandra observations show the SNR clearly in X-rays, although the southern rim is much fainter than the rest of the SNR. We have measured and adopted the full extent of the SNR shown in X-rays, about 33.5 pc, similar as the size measured by Maggi et al. (2016), which is larger than those reported by De2010 and Bo2017.

DEM L238.- Comparing $\mathrm{H} \alpha$ and Chandra images, the X-ray emitting region is larger than the optical shell. We adopt the full extent of the SNR, $47.5 \mathrm{pc}$.

Honeycomb.- The Honeycomb SNR is near the 30 Doradus complex, and to the south of the superbubble 30 Dor C. This region has a very complex star formation history and chaotic nebular morphology. The lack of bright ionized gas region suggests an evolved environment with low ISM densities. The optical morphology of the SNR is very irregular, consisting of many cells instead of a simple shell (Chu et al. 1995; Meaburn et al. 2010), leading to large uncertainty in the determination of SNR size. For the Honeycomb SNR, we have used both sizes of De2010 (15 pc) and Ba2010 $(25.5 \mathrm{pc})$ in the $L_{X}$ - size plot to illustrate the uncertainty in the size.

N157B.- The environment of N157B in $\mathrm{H} \alpha$ is very complex because this SNR is superposed on the HII region of the OB association LH99 (Chu 1997), and dissected by a foreground dark cloud. The most reliable measurement of the SNR size is through the analysis of gas kinematics using long-slit high-dispersion spectroscopic observations, $25 \times 18$ pc (Chu et al. 1992). This SNR boundary has been confirmed by sharp filaments revealed by HST images as shown in Figure 5. The size 21.8 pc given by De2010 is taken from Chu et al. (1992).

DEM L299.- This SNR is inside a large optical shell. The size reported by De2010 corresponds to the large optical shell. The X-ray emission actually extends from the shell cavity to the southwest, indicating an outflow. The [S II] $] / \mathrm{H} \alpha$ ratio is enhanced in the shell structure and in the superposed filaments of supergiant shell LMC-2. The SNR is clearly in a very complex environment. We include all the diffuse X-ray emission region and [S II] enhanced filaments, and measure a size of $100 \times 50$ pc. A smaller SNR size, $\sim 55$ pc, has been reported by Warth et al. (2014) and Maggi et al. (2016) based on the diffuse X-ray emission and a surrounding [S II]-enhanced filament. The large discrepancy between these two size measurements illustrate the difficulty in determining SNR sizes in a complex environment confused by other energetic feedback processes from massive stars. We adopt both 73.5 and 56.5 pc in Table 1 (Appendix C) and Figure 2.

J0550-6823.- While the diameter of the optical shell is only $\sim 68 \mathrm{pc}$, there is X-ray and radio emission extending over $90 \mathrm{pc}$ in the east-west direction; therefore, we adopt the size of $90 \times 68$ pc by Bozzetto et al. (2012). 


\section{SIZES AND X-RAY LUMINOSITIES OF LMC SNRS}

Table 1. Sizes and X-ray luminosities of LMC SNRs

\begin{tabular}{|c|c|c|c|c|c|c|c|}
\hline SNR J2000 a & Other Name & $\begin{array}{c}\text { Size (Ba2010) } \\
\text { (pc) }\end{array}$ & $\begin{array}{c}\text { Size (De2010) } \\
\text { (pc) }\end{array}$ & $\begin{array}{c}\text { Size (Bo2017) } \\
(\mathrm{pc})\end{array}$ & $\begin{array}{l}\text { Large Discrepancy }{ }^{\mathrm{b}} \\
\qquad(>16 \%)\end{array}$ & $\begin{array}{c}\text { Adopted Size } \\
\text { (pc) }\end{array}$ & $\begin{array}{c}L_{\mathrm{X}}{ }^{\mathrm{c}} \\
\left(10^{35} \mathrm{ergs} / \mathrm{s}\right)\end{array}$ \\
\hline J0448-6700 & [HP99] 460 & 55.0 & 59.2 & 60.8 & & 60.8 & 0.46 \\
\hline J0449-6920 & - & 33.2 & 30.0 & 28.8 & & 28.8 & 0.07 \\
\hline J0450-7050 & SNR 0450-70.9 & 89.2 & 97.5 & 109.4 & & 109.4 & 0.59 \\
\hline J0453-6655 & $\mathrm{SNR}$ in N4 & 63.0 & 64.5 & 60.6 & & 60.6 & 1.17 \\
\hline J0453-6829 & SNR 0453-68.5 & 30.0 & 30.0 & 30.4 & & 30.4 & 13.85 \\
\hline J0454-6713 & SNR 0454-67.2 & 44.2 & 37.5 & 32.5 & & 32.5 & 1.58 \\
\hline J0454-6626 & N11L & 21.8 & 18.0 & 20.4 & & 20.4 & 0.63 \\
\hline J0455-6839 & N86 & 87.0 & 75.0 & 61.5 & Y & $61.5-75.0$ & 1.42 \\
\hline J0459-7008 & N186D & 37.5 & 36.8 & 29.0 & Y & 36.8 & 1.09 \\
\hline J0505-6753 & DEM L71 & 18.0 & 20.2 & 18.6 & & 18.6 & 44.59 \\
\hline J0505-6802 & $\mathrm{N} 23$ & 27.8 & 15.0 & 23.6 & Y & 23.6 & 26.25 \\
\hline J0506-6541 & DEM L72 & 102.0 & 83.2 & 96.2 & & 96.2 & 0.53 \\
\hline J0506-7026 & [HP99] 1139 & 82.5 & - & 42.5 & & 42.5 & 1.44 \\
\hline J0508-6902 & [HP99] 791 & - & - & 67.0 & & 67.0 & 0.37 \\
\hline J0508-6830 & - & - & - & 30.8 & & 30.8 & 0.09 \\
\hline J0509-6844 & N103B & 7.0 & 7.5 & 7.0 & & 7.0 & 51.7 \\
\hline J0509-6731 & SNR 0509-67.5 & 7.25 & 8.4 & 7.6 & & 7.6 & 16.51 \\
\hline J0511-6759 & - & - & - & 55.5 & & 55.5 & 0.16 \\
\hline J0512-6707 & [HP99] 483 & - & - & 12.5 & & 12.5 & 0.09 \\
\hline J0513-6912 & DEM L109 & 53.8 & 57.8 & 55.6 & & 55.6 & 0.51 \\
\hline J0514-6840 & - & - & - & 55.0 & & 55.0 & 0.4 \\
\hline J0517-6759 & - & - & - & 66.8 & & 66.8 & 0.24 \\
\hline J0518-6939 & N120 & 33.5 & 21.8 & 23.4 & & 23.4 & 0.88 \\
\hline J0519-6902 & SNR 0519-69.0 & 7.8 & 8.2 & 8.6 & & 8.6 & 34.94 \\
\hline J0519-6926 & SNR 0520-69.4 & 43.5 & 33.8 & 31.2 & & 31.2 & 2.69 \\
\hline J0521-6543 & DEM L142 & - & 40.5 & 34.5 & & 34.5 & - \\
\hline J0523-6753 & SNR in N44 & 57.0 & 52.5 & 57.5 & & 57.5 & 0.9 \\
\hline J0524-6624 & DEM L175a & 58.5 & 51.8 & 36.25 & & 36.2 & - \\
\hline J0525-6938 & N132D & 28.5 & 26.2 & 25.5 & & 25.5 & 315.04 \\
\hline J0525-6559 & N49B & 42.0 & 36.0 & 38.8 & & 38.8 & 38.03 \\
\hline J0526-6605 & N49 & 21.0 & 21.0 & 18.8 & & 18.8 & 64.37 \\
\hline J0527-6912 & SNR 0528-69.2 & 36.8 & 35.2 & 35.0 & & 35.0 & 1.99 \\
\hline J0527-6550 & DEM L204 & 75.8 & 67.5 & 76.2 & & 76.2 & - \\
\hline J0527-6714 & SNR 0528-6716 & - & - & 54.0 & & 54.0 & 0.58 \\
\hline
\end{tabular}

Table 1 continued 
Table 1 (continued)

\begin{tabular}{|c|c|c|c|c|c|c|c|}
\hline SNR J2000 ${ }^{a}$ & Other Name & $\begin{array}{c}\text { Size }(\text { Ba2010) } \\
\text { (pc) }\end{array}$ & $\begin{array}{c}\text { Size (De2010) } \\
\text { (pc) }\end{array}$ & $\begin{array}{c}\text { Size }(\text { Bo2017) } \\
\text { (pc) }\end{array}$ & $\begin{array}{c}\text { Large Discrepancy }{ }^{\mathrm{b}} \\
\qquad(>16 \%)\end{array}$ & $\begin{array}{l}\text { Adopted Size } \\
\text { (pc) }\end{array}$ & $\begin{array}{c}L_{\mathrm{X}}^{\mathrm{c}} \\
\left(10^{35} \mathrm{ergs} / \mathrm{s}\right)\end{array}$ \\
\hline J0527-7104 & [HP99] 1234 & 49.0 & - & 70.2 & & 70.2 & 0.25 \\
\hline J0528-6727 & DEM L205 & - & - & 55.0 & & 55.0 & 0.21 \\
\hline J0529-6653 & DEM L214 & 25.0 & - & 33.1 & & 33.1 & 1.04 \\
\hline J0530-7008 & DEM L218 & 53.2 & 47.2 & 49.4 & & 49.4 & 0.72 \\
\hline J0531-7100 & N206 & 48.0 & 45.0 & 45.0 & & 45.0 & 2.55 \\
\hline J0532-6732 & SNR 0532-67.5 & 63.0 & 67.5 & 45.0 & $\mathrm{Y}$ & 67.5 & 2.48 \\
\hline J0533-7202 & - & - & - & 45.0 & & 45.0 & 0.57 \\
\hline J0534-6955 & SNR 0534-69.9 & 28.5 & 23.2 & 28.8 & $\mathrm{Y}$ & 33.5 & 6.33 \\
\hline J0534-7033 & DEM L238 & 45.0 & 40.5 & 47.5 & $\mathrm{Y}$ & 47.5 & 1.55 \\
\hline J0535-6916 & SN 1987A & 0.5 & $>1.5$ & 0.45 & & 0.45 & 27.39 \\
\hline J0535-6602 & N63A & 16.5 & 19.5 & 18.5 & & 18.5 & 185.68 \\
\hline J0535-6918 & Honeycomb & 25.5 & 15.0 & 18.8 & $\mathrm{Y}$ & $15.0-25.5$ & 0.4 \\
\hline J0536-6735 & DEM L241 & 33.8 & 36.0 & 34.0 & & 34.0 & 3.84 \\
\hline J0536-7039 & DEM L249 & 45.0 & 37.5 & 39.2 & & 39.2 & 1.43 \\
\hline J0536-6913 & SNR 0536-69.2 & 120 & - & 16.5 & & 16.5 & 0.22 \\
\hline J0537-6628 & DEM L256 & 51.0 & 48.0 & 46.9 & & 46.9 & 0.32 \\
\hline J0537-6910 & N157B & 25.5 & 21.8 & 31.5 & $\mathrm{Y}$ & 21.8 & 15.0 \\
\hline J0540-6944 & SNR in N159 & 19.5 & 27.0 & 26.2 & & 26.2 & 0.43 \\
\hline J0540-6920 & SNR 0540-69.3 & 15.0 & 18.0 & 15.6 & & 15.6 & 87.35 \\
\hline J0541-6659 & [HP99] 456 & - & - & 71.5 & & 71.5 & 0.77 \\
\hline J0543-6858 & DEM L299 & 79.5 & 73.5 & 56.5 & $\mathrm{Y}$ & $56.5-73.5$ & 1.68 \\
\hline J0547-6943 & DEM L316B & 21.0 & 46.5 & 45.0 & & 45.0 & 1.47 \\
\hline J0547-6941 & DEM L316A & 14.0 & 30.0 & 30.0 & & 30.0 & 1.26 \\
\hline J0547-7025 & SNR 0548-70.4 & 25.5 & 28.5 & 28.1 & & 28.1 & 2.94 \\
\hline J0550-6823 & - & 78.0 & 65.2 & 81.9 & $\mathrm{Y}$ & 81.9 & 1.22 \\
\hline
\end{tabular}

$a_{\text {The }} 59$ confirmed SNRs listed in Maggi et al. (2016).

${ }^{b}$ Size(De2010)/Size(Bo2017) $>1.16$ or $<0.86$.

${ }^{c}$ Taken from Maggi et al. (2016). 